\title{
Barrier-layer-mediated Electron Transfer from Semiconductor Electrodes to Molecules in Solution. Sensitivity of Mechanism to Barrier Layer Thickness
}

\author{
Jason R. Avila, ${ }^{\dagger,}$ Michael J. Katz, Omar K. Farha, ${ }^{\dagger, \dagger}$ and Joseph T. Hupp ${ }^{\dagger}, *$ \\ †Department of Chemistry, Northwestern University, 2145 Sheridan Road Evanston, Illinois 60208, United States \\ ${ }^{\ddagger}$ King Abdulaziz University, Faculty of Science, Department of Chemistry, Jeddah, Saudi Arabia \\ ${ }^{+}$Department of Chemistry, Memorial University of Newfoundland, St. John's, NL A1B 3X7 Canada
}

\section{Table of Contents}

SI-1: Experimental details

SI-2: Lifetime analysis of tunneling-hopping supplemental figures

SI-3: Electrochemical Impedance Spectroscopy analysis of tunneling-hopping transition

SI-4: References

\section{SI-1 - Experimental}

All atomic layer deposition materials were purchased from Strem Chemicals, all other chemicals were purchased from Sigma Aldrich. Depositions were carried out in a Savannah 100 ALD reactor with flowing $\mathrm{N}_{2}$ as a carrier gas (Cambridge Nanotech, Inc.). Metal-organic ALD precursors tested in this study included tetrakis(dimethylamido)tin (TDMASn) and tetra(isopropyl)titatium (TTIP). Both precursors used water as an oxygen source. All ALD reactions were done at $150{ }^{\circ} \mathrm{C}$, with TDMASn and TTIP precursor reservoirs set at 40 and $80{ }^{\circ} \mathrm{C}$, respectively. TDMASn and TTIP were pulsed for $0.15 \mathrm{~s}$ and water was pulsed for $15 \mathrm{~ms}$. All precursor pulses were followed by a $30 \mathrm{~s}$ purge under flowing N2. Thickness of $\mathrm{SnO}_{2}$ and $\mathrm{TiO}_{2}$ grown by ALD were confirmed using ellipsometry (A.J.A. Woolam Co. M2000) on a Si witness substrate during each run.

Working electrodes used in this study were grown on FTO glass that was sonicated in acetone, isopropanol and soapy water, followed by a 30 minute ozone treatment to remove all surface contamination. $30 \mathrm{~nm}$ of $\mathrm{SnO}_{2}$ was then deposited on FTO by ALD that was then annealed in air at 450 ${ }^{\circ} \mathrm{C}$ for 30 minutes, ramping up from room temperature for 2 hours. $\mathrm{A} \sim 1 \mathrm{~cm}^{2}$ area of the $\mathrm{SnO}_{2}$ was then masked off using kapton tape to ensure similar surface area between working electrodes. The working electrodes were then measured electrochemically after every 3 cycles of $\mathrm{TiO}_{2}(\sim 1 \AA$ thick$)$. Once measured, the films were washed with acetonitrile before depositing additional layers of $\mathrm{TiO}_{2}$. A 15 minutes purge time before $\mathrm{TiO}_{2}$ deposition was used to ensure evaporation of any surface absorbed solvent.

Electrochemical analysis was done using a Solartron analytical modulab instrument, equipped with a potentiostat and frequency analyzer module. A three electrode set up was utilized using a platinum mesh counter electrode and a saturated $\mathrm{Ag} / \mathrm{AgCl}$ reference electrode. The electrolyte solution consisted of 0.6 $\mathrm{M}$ butylmethylimidazoliumiodide and $0.03 \mathrm{M} \mathrm{I}_{2}$ in acetonitrile. Open circuit voltage decay (OCVD) measurements were achieved by applying a constant potential at $-300 \mathrm{mV}$ vs. $\mathrm{Ag} / \mathrm{AgCl}$ for $30 \mathrm{~seconds}$ 
before measuring the potential as a function of time at open circuit. For electrochemical impedance spectroscopy (EIS), a DC voltage was kept at $-300 \mathrm{mV}$ vs. $\mathrm{Ag} / \mathrm{AgCl}$ with a $10 \mathrm{mV}$ oscillating voltage with a frequency range of $0.5 \mathrm{MHz}$ to $0.05 \mathrm{~Hz}$. Density of state (DOS) analysis of ALD grown $\mathrm{TiO}_{2}$ used a $50 \mathrm{~nm}$ film of $\mathrm{TiO}_{2}$ grown on FTO. Electrochemical impedance spectroscopy was done as above but at every $100 \mathrm{mV}$ between 0 to $-700 \mathrm{mV}$ vs. $\mathrm{Ag} / \mathrm{AgCl}$. The capacitance (fit to a simple $\mathrm{RC}$ circuit) was converted to DOS using a similar method published by Bisquert and co-workers. ${ }^{1}$ See previous work for synthesis of shuttle molecules (cobalt coordination complexes). ${ }^{2}$ For these studies, $0.01 \mathrm{M}$ of the Co(III) form of the shuttle molecule was used along with $0.2 \mathrm{M} \mathrm{LiClO}_{4}$ or $\mathrm{LiPF}_{6}$ as supporting electrolyte.

\section{SI-2 - Lifetime analysis of tunneling-hopping supplemental figures}

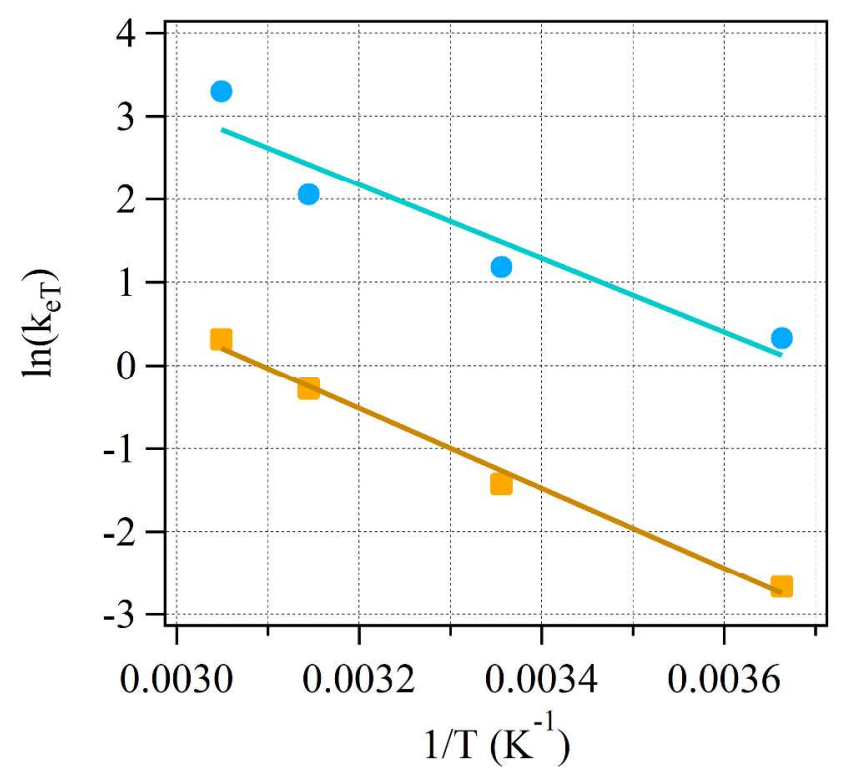

Figure S1: The $\operatorname{In}\left(k_{e T}\right)$ as a function of the inverse of electrolyte temperature for $5.5 \mathrm{~nm}$ of $\mathrm{ALD} \mathrm{TiO}_{2}$ as-deposited (blue circles) and after annealing at $450{ }^{\circ} \mathrm{C}$ (yellow squares) on $\mathrm{SnO}_{2}$ flat films.

Figure S1 shows the change in electron transfer rate as a function of the inverse of the electrolyte solution temperature at $0,25,45$, and $55^{\circ} \mathrm{C}$. The Arrhenius relationship between the electron transfer rate (keT) and the temperature yields the linear traces shown in Figure S1. The activation energy, determined from the slope, is 36 and $40 \mathrm{~kJ} / \mathrm{mol}$ for the as-deposited (blue circles) and annealed $\mathrm{TiO}_{2}$, respectively. 

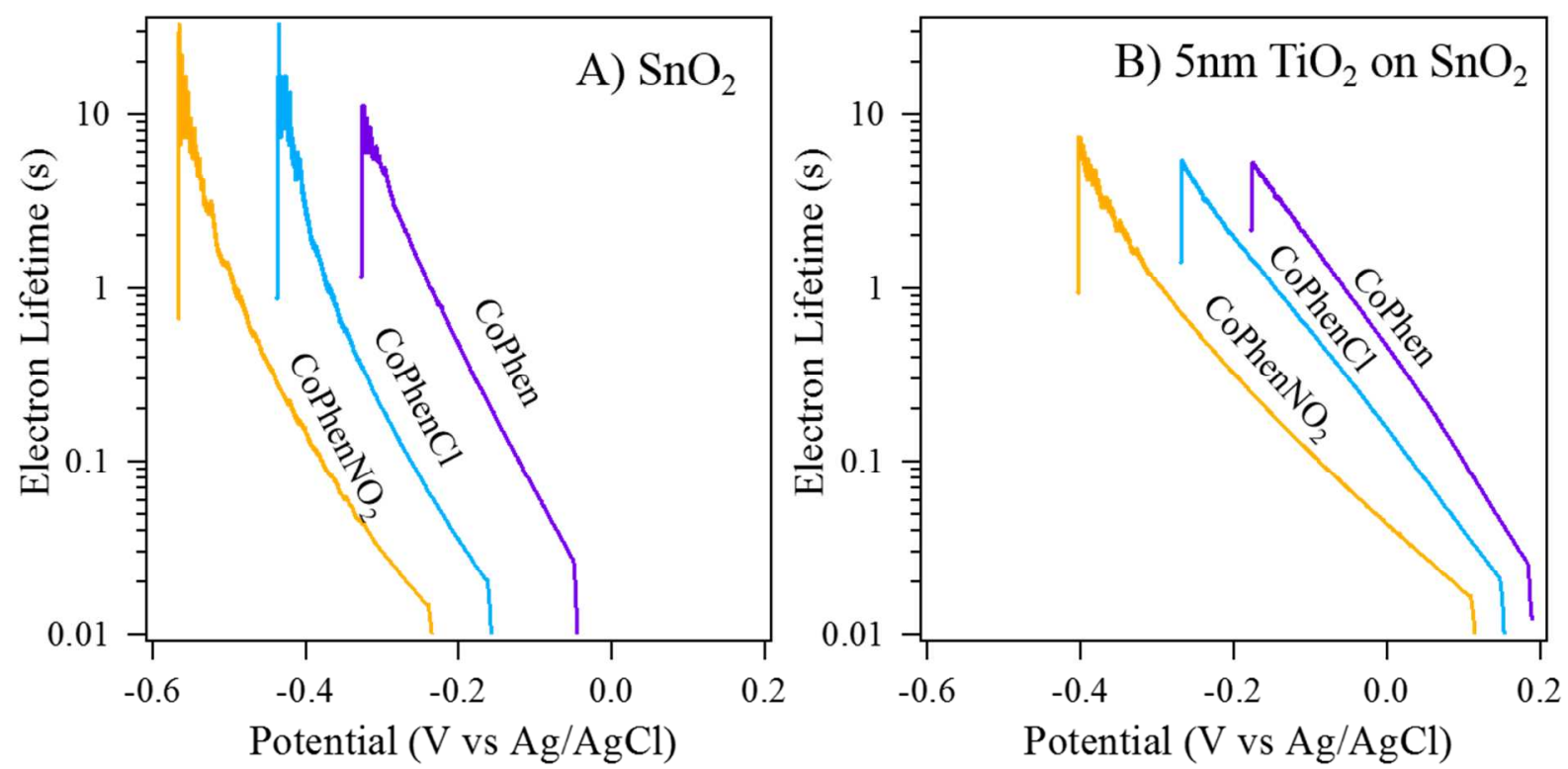

Figure S3: Electron lifetime as a function of potential determined by OCVD measurements of A) bare $\mathrm{SnO}_{2}$ and B) $5 \mathrm{~nm}$ of $\mathrm{TiO}_{2}$ on $\mathrm{SnO}_{2}$ using various tris-phenanthroline complexes of cobalt (III).

Similar to previous studies, ${ }^{2}$ substituents on polypyridine ligands were used to vary the formal potential of the solution-phase electron acceptor. If $\mathrm{ALD} \mathrm{TiO}_{2}$ serves chiefly as a tunneling barrier, rates of back-ET to a series of acceptors should increase with increasing electrochemical driving force (i.e. Marcus-type behavior should be observed). Conversely, if ET from the electrode to the molecular acceptor species is a fast process, with access to intermediate electronic states within the $\mathrm{TiO}_{2}$ layer being the slow step in a multi-step process assess, rates should be insensitive to the overall electrochemical driving force. With this question in mind, we examined back-ET, via OCVD, with three members of an isostructural series of molecular acceptors. if the ET by trap hopping follows Marcus normal ET behavior. The first electron acceptor, tris-phenanthroline cobalt(III/II) (CoPhen), has the least positive formal potential, and thus, is subject to the smallest thermodynamic driving force when a $\mathrm{SnO}_{2}$ electrode is the electron donor. Adding one chloro substituent to each ligand $(\mathrm{CoPhenCl})$ shifts the potential more positive by several tens of millivolts, while introduction one nitro group for each ligand instead $\left(\mathrm{CoPhenNO}_{2}\right)$ yields a larger positive shift and a correspondingly large (more favorable) driving force for back-ET. Figure S3 shows that back-ET from both bare $\mathrm{SnO}_{2}$ and $\mathrm{TiO}_{2}$-coated electrodes is responsive to the formal potential of the electron acceptor, with the ordering of rates being: $\mathrm{CoPhenNO}_{2}>\mathrm{CoPhenCl}>\mathrm{CoPhen}$.

\section{SI-3 - Electrochemical Impedance Spectroscopy (EIS) assessment of tunneling-hopping transition}

For initial EIS measurements, the DC potential for the working electrode was fixed at $-300 \mathrm{mV}$ vs $\mathrm{Ag} / \mathrm{AgCl}$ (i.e. the same potential as used to initiate OCVD experiments). The cell resistance and capacitance was then evaluated as a function of barrier-layer thickness. Figure S3 shows the Nyquist plot (A) and phase plot (B) of a cell featuring a $\mathrm{SnO}_{2}$ working electrode, with out and with varying amounts of ALD $\mathrm{TiO}_{2}$. From the Nyquist behavior (Figure S3-A), there are clear increases in resistance with increasing $\mathrm{TiO}_{2}$ layer thickness. The resistance elements, represented by the wide arc of the Nyquist plot, initially increase with layer thickness, but are only slightly changed once the barrier thickness exceeds $\sim 10 \AA$. If the observed resistance is interpreted as a charge-transfer resistance, the observations are consistent with the conclusions drawn from OCVD measurements. 
The Bode plot (Figure S3-B), showing changes in phase as a function of frequency, features a single peak when the $\mathrm{TiO}_{2}$-based barrier layer is absent, but two peaks when it is present. A consistent picture emerges if we assume that the single peak in Figure S3-B for bare $\mathrm{SnO}_{2}$ (grey crosses) derives from the electrode/solution charge-transfer reaction. The peak shifts to lower frequency as barrier layers of increasing thickness are introduced, indicating greater charge-transfer resistance and, therefore, slower electron-transfer. Further increases in barrier thickness, however, elicit no additional increase in chargetransfer resistance, supporting the assignment of the peak to electrode/solution charge-transfer mirrors, and mirroring the ET kinetics measured by OCVD. In the presence of a barrier layer, a second peak, at higher frequency, is observed. We tentatively assign this peak to hopping between localized titanium sites.
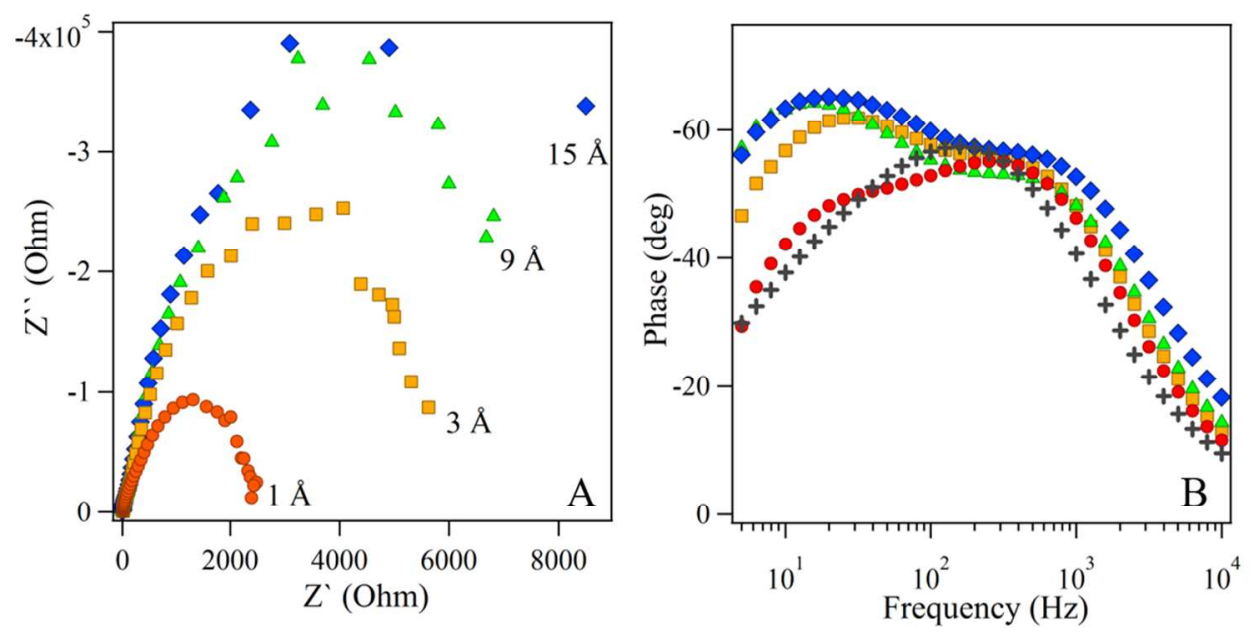

Figure S3: Nyquist plot (A) and Bode plot (bottom) of 1 (red circles), 3 (orange squares), 9 (green triangles), and 15 (blue diamonds) A of $\mathrm{TiO}_{2}$ grown by $\mathrm{ALD}$ on $30 \mathrm{~nm}$ flat $\mathrm{SnO}_{2}$ films. On the bode plot (B) grey crosses correspond to bare $\mathrm{SnO}_{2}$. It should be noted that, for graphical clarity, the Nyquist plot for bare $\mathrm{SnO}_{2}$ was omitted.

With the hypothesized two-stage electron transport pathway, we can fit the impedance results to a simple $\mathrm{RC}$ circuit shown in Figure S4-insert. The double RC circuit shows two resistance elements which includes $\mathrm{R}_{\mathrm{CT}}$, electrode/redox couple charge transfer which includes electron tunneling, and $\mathrm{R}_{\text {hop }}$, charge transfer to/ between holes in the $\mathrm{TiO}_{2}$ blocking layer. In this picture, $\mathrm{C}_{\mu}$ and $\mathrm{C}_{\text {hop }}$ represent, respsectively, the capacitances of the space charge layer and of mid-gap states. The series resistance, $\mathrm{R}_{\mathrm{s}}$, is assumed to arise from the working electrode sheet-resistance and/or the solution resistance. From the fit values in Figure S4, there is a clear change in charge-transfer resistance as the thickness of the $\mathrm{TiO}_{2}$ film increases. When the film reaches a thickness of $\sim 10 \AA$, there is a plateau indicating little additional resistance with increasing thickness of $\mathrm{TiO}_{2}$. The behavior observed here is consistent with that illustrated in Figure 3, where the distance dependence of single-step barrier tunneling serves to modulate the kinetics. For thicker layers, a multi-step process, signified by $\mathrm{R}_{\text {hop }}$ and corresponding to hopping between sites within the barrier, serves to arrest the decrease in overall rate with increasing barrier thickness. 


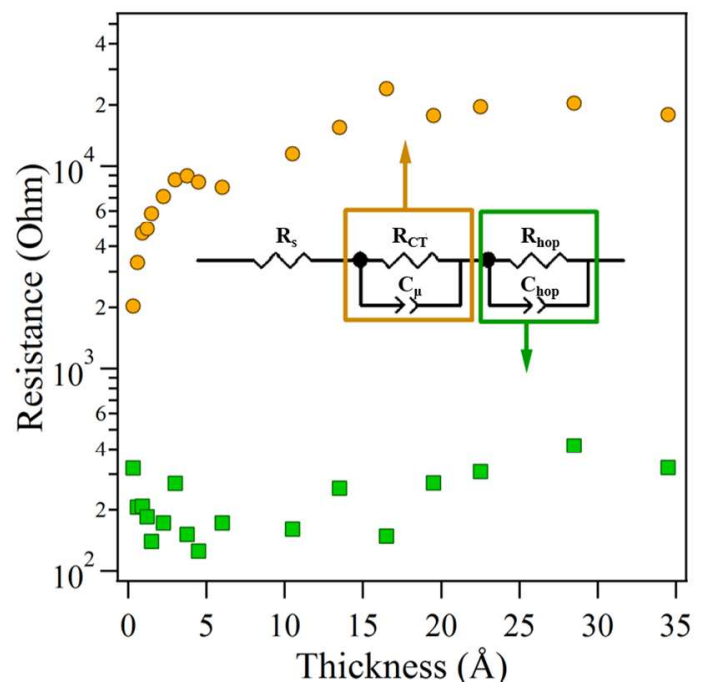

Figure S4: Fits to EIS fits to the charge transfer resistance at the surface ( $R_{C T}$-orange circles) and to the $\mathrm{TiO}_{2}$ film $\left(R_{\text {hop }}\right.$ - green squares). The RC circuit shown in the inset was used as a model for this system.

Figure S5 illustrates the effect of $\mathrm{TiO}_{2}$ film annealing the on the impedance spectrum. As in Figure $\mathrm{S} 3$, the electrode featuring an as-deposited film yields a two-peak Bode plot; see Figure S5 - blue circles). Film annealing at $450{ }^{\circ} \mathrm{C}$ transforms the plot to one featuring only a single peak (albeit broadened compared to those for the as-deposited electrode). The inset shows Nyquist plots, where the data have been fit to a simplified circuit containing only a single resistor and a single capacitor. The plots indicate a $c a$. 17-fold increase in resistance following film annealing, finding consistent with the decreases in $k_{e T}$ that accompany annealing.

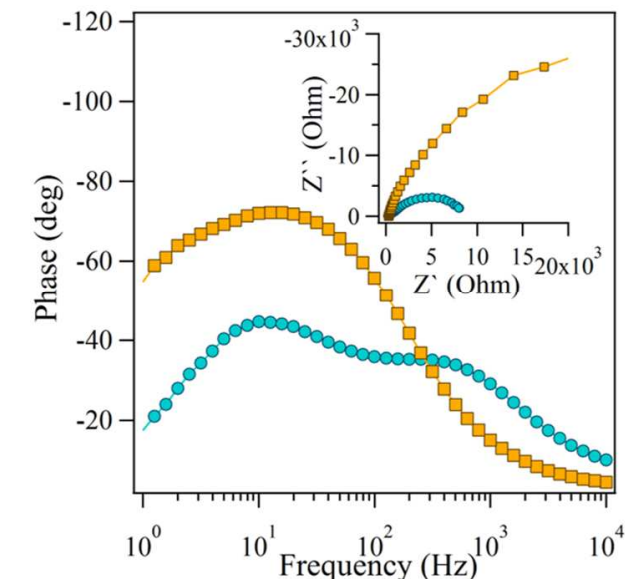

Figure S5: Bode plot and Nyquist plot (insert) of $5.5 \mathrm{~nm}$ of $\mathrm{TiO}_{2}$ on $\mathrm{SnO}_{2}$ before (blue circles) and after annealing (orange squares). 


\section{SI-4 - References}

1. Bisquert, J.; Vikhrenko, V. S., Interpretation of the Time Constants Measured by Kinetic Techniques in Nanostructured Semiconductor Electrodes and Dye-Sensitized Solar Cells. J. Phys. Chem. $B$ 2004, 108, 2313-2322.

2. DeVries, M. J.; Pellin, M. J.; Hupp, J. T., Dye-sensitized solar cells: driving-force effects on electron recombination dynamics with cobalt-based shuttles. Langmuir : The ACS Journal of Surfaces and Colloids 2010, 26, 9082-7. 